\title{
SDOCT Thickness Measurements of Various Retinal Layers in Patients with Autosomal Dominant Optic Atrophy due to OPA1 Mutations
}

\author{
Andrea M. Schild, ${ }^{1}$ Tina Ristau, ${ }^{2}$ Julia Fricke, ${ }^{1}$ Antje Neugebauer, ${ }^{1}$ Bernd Kirchhof, ${ }^{1}$ \\ Srinivas R. Sadda, ${ }^{3}$ and Sandra Liakopoulos ${ }^{2}$ \\ ${ }^{1}$ Department of Ophthalmology, University Hospital of Cologne, 50924 Cologne, Germany \\ ${ }^{2}$ Cologne Image Reading Center, Department of Ophthalmology, University Hospital of Cologne, 50924 Cologne, Germany \\ ${ }^{3}$ Doheny Image Reading Center, Doheny Eye Institute, 1450 San Pablo Street, Los Angeles, CA 90033, USA
}

Correspondence should be addressed to Andrea M. Schild; a.schild@gmx.de

Received 8 April 2013; Revised 16 July 2013; Accepted 19 July 2013

Academic Editor: Marcela Votruba

Copyright (c) 2013 Andrea M. Schild et al. This is an open access article distributed under the Creative Commons Attribution License, which permits unrestricted use, distribution, and reproduction in any medium, provided the original work is properly cited.

\begin{abstract}
Purpose. To specify thickness values of various retinal layers on macular spectral domain Optical Coherence Tomography (SDOCT) scans in patients with autosomal dominant optic atrophy (ADOA) compared to healthy controls. Methods. SDOCT volume scans of 7 patients with ADOA (OPA-1 mutation) and 14 healthy controls were quantitatively analyzed using manual grading software. Mean thickness values for the ETDRS grid subfields 5-8 were calculated for the spaces neurosensory retina, retinal nerve fiber layer (RNFL), ganglion cell layer (GCL), a combined space of inner plexiform layer/outer plexiform layer/inner nuclear layer (IPL+INL+OPL), and a combined space of outer nuclear layer/photoreceptor layers (ONL+PL). Results. ADOA patients showed statistically significant lower retinal thickness values than controls $(P<0.01)$. RNFL $(P<0.001)$ and GCL thicknesses $(P<0.001)$ were significantly lower in ADOA patients. There was no difference in IPL+INL+OPL and in ONL+PL thickness. Conclusion. Manual subanalysis of macular SDOCT volume scans allowed detailed subanalysis of various retinal layers. Not only RNFL but also GCL thicknesses are reduced in the macular area of ADOA patients whereas subjacent layers are not involved. Together with clinical findings, macular SDOCT helps to identify patients with suspicion for hereditary optic neuropathy before genetic analysis confirms the diagnosis.
\end{abstract}

\section{Introduction}

Autosomal dominant optic atrophy (ADOA; OMIM 165500) is caused by a mutation in the OPA1 gene $[1,2]$. The OPA1 gene encodes a dynamin-related GTPase that plays an important role in mitochondrial fusion processes, stabilization of cristae, and cytochrome $c$ sequestration $[3,4]$. ADOA generally presents with mild visual impairment in the first decade of life. Over years, ADOA leads to a slow continuous decrease in visual acuity (VA), which varies among individuals. Finally, visual loss ranges from a very mild affection to severe impairment $[5,6]$.

Visual fields usually show central, paracentral, or centrocecal scotomas. Atrophy of the optic disc may not be visible in the beginning, but with progression of the disease the optic disc typically shows a temporal or diffuse pallor [7]. Histological examinations have revealed a loss in retinal ganglion cells especially in the macular area and in the papillomacular bundle [8]. Loss in vision has been demonstrated to correspond to a reduction of the retinal nerve fiber layer (RNFL) on Stratus optical coherence tomography (OCT) [9]. A reduction of RNFL and GCL thicknesses is also seen in patients with optic atrophy of other origin $[10,11]$.

Spectral domain OCT (SDOCT) instruments allow improved visualization of various retinal layers with almost histological quality. The technology of SDOCT is noninvasive, fast, and easy to apply even in young children [12]. 
The aim of this study was to analyze thickness values of various retinal layers on SDOCT volume scans of the posterior pole using manual OCT grading software in patients with ADOA associated with OPA1 mutations compared to healthy controls.

\section{Materials and Methods}

Seven right eyes of 7 unrelated patients with genetically confirmed ADOA with heterozygous mutation in the OPA1 gene were compared to 14 right eyes of 14 healthy controls matching in age, refractive error, and gender.

SDOCT (Spectralis OCT, Heidelberg Engineering, Germany) derived volume scans of the macular area $(15 \times$ $20^{\circ}$ ) and best corrected VA were retrospectively collected for patients and controls. Volume scans were quantitatively analyzed using computer-assisted manual grading software (3D-OCTOR), which facilitates manual delineation of various retinal layers. The software has been validated in previous reports [13-15]. After drawing the required layers for all spaces with a computer mouse, the software generates thickness and volume values for each of the nine subfields of the Early Treatment of Diabetic Retinopathy Study (ETDRS) grid.

After the ETDRS grid was manually centered on the fovea, mean thickness values for the inner circle of the ETDRS grid (fields 5-8, $3 \mathrm{~mm}$ diameter) were calculated for the following spaces: neurosensory retina, retinal nerve fiber layer (RNFL), ganglion cell layer (GCL), the combined space of inner and outer plexiform layers and inner nuclear layer (IPL+INL+OPL), and the combined space of outer nuclear layer and photoreceptor layer (ONL+PL) (Figures 1 and 2). The foveal central subfield (FCS, $1 \mathrm{~mm}$ diameter) was not included in the analysis due to the variability of the foveal depression, which may influence thickness measurements.

Statistical analysis was performed using commercially available software (Sigma Plot for Windows version 11.0 Systat Software Inc., Germany). The Mann-Whitney $U$ Test was used to compare thickness values between patients and controls. Intraindividual thickness values of the subfields 5 to 8 were compared using ANOVA on ranks analysis. For statistical analysis, Snellen VA was converted to logarithm of the minimum angle of resolution (logMAR). The correlation between OCT parameters and VA was assessed using Spearman's rank correlation coefficient and regression analysis. This study adhered to the tenets set forth in the Declaration of Helsinki.

\section{Results}

Four male and 3 female ADOA patients were included in the study. The mean age was 25 years ( $\min 4 ; \max 47)$. VA ranged from $20 / 800$ to $20 / 40$. Two patients reported progressive visual loss since early childhood. In 5 patients, the diagnosis was made by chance during a routine check by an outpatient ophthalmologist. Clinical and genetic data of all patients are summarized in Table 1.

Fourteen right eyes of 14 healthy individuals were included in the study as controls. The mean age was 27 years ( $\min 3$; $\max 54)$. Refractive error ranged from $-6.0 \mathrm{dpt}$ to $+2.3 \mathrm{dpt}$ (spherical equivalent) comparable to the group of patients. VA was 20/20 in all cases. Visual fields and disc appearance were unremarkable. A summary of median thickness values of all subfields is provided in Table 2 .

Median thickness of the neurosensory retina was statistically significant lower in ADOA patients compared to healthy controls $(P<0.01)$. Subanalysis of retinal layers revealed that RNFL thickness $(P<0.001)$ as well as GCL thickness $(P<0.001)$ measurements were significantly lower in patients with ADOA for each of the ETDRS subfields 5, 6,7 , and 8 . However, there was no difference in ONL+PR thickness values as well as in IPL+INL+OPL thickness values between groups. The differences in thickness values between patients and controls were not only found in adult patients but also in the two children of 4 and 11 years of age with recent diagnosis of the disease.

Intraindividual subanalysis of the mean thickness values for subfields 5 to 8 revealed significant difference between the nasal, temporal, inferior, and superior subfields in both groups. In ADOA patients, RNFL thickness appeared to show the lowest values in subfield 8 (temporal) and a statistically significant difference was detected only for RNFL thickness of subfield 8 compared to subfield $5(P=0.04)$. For all other spaces, there was no difference between subfields.

In healthy controls, neurosensory retina was significantly thinner in subfield 8 than in subfield $5(P<0.001)$, subfield 6 $(P=0.006)$, and subfield $7(0.02)$, RNFL in subfield 8 was significantly thinner compared to subfield $5(P<0.001)$, subfield $6(P<0.001)$, and subfield $7(P<0.001)$, and subfield 6 was significantly thinner compared to subfield 5 $(P=0.01)$, and subfield $7(P<0.001)$. The combined space of INL+OPL+IPL was also significantly thinner in subfield 8 than in subfield $5(P=0.03)$, subfield $6(P=0.01)$ and subfield 7 (0.02). GCL and ONL+PR showed no difference between subfields.

In our study group of ADOA patients, neither regression analysis nor Spearman's analysis showed a significant correlation between VA or age and GCL thickness or RNFL thickness.

\section{Discussion}

High resolution SDOCT images and manual subanalysis of retinal layers allowed detailed quantitative analysis of various inner and outer retinal layers within the macular region in ADOA patients. We could confirm that inner retinal layers are primarily affected in ADOA patients and demonstrate that thinning of inner retinal layers is explained by a reduction in RNFL thickness as well as GCL thickness without any effect on IPL+INL+OPL thickness. The results from our study are in line with marked loss of GCL and retinal ganglion cell axons in the macula as previously documented in postmortem histopathological studies of patients with ADOA [8]. Lower thickness values of inner retinal layers were even evident in young patients newly diagnosed with the disease.

Recent OCT studies mainly focused on measurements of peripapillary RNFL. Milea et al. [16] reported a gradual 


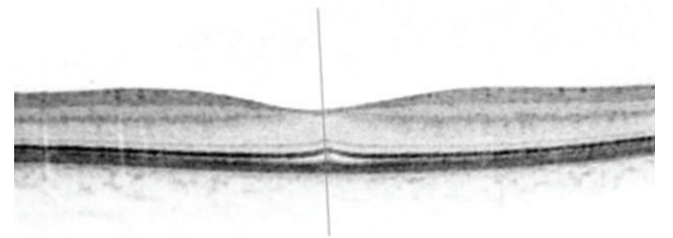

(a)

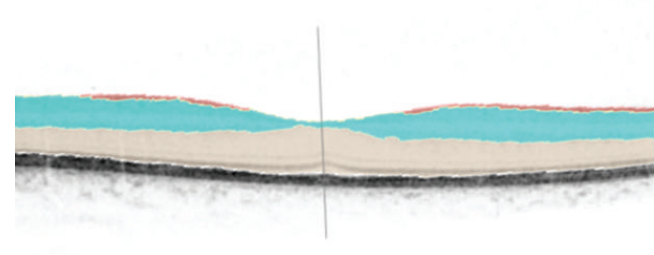

(b)

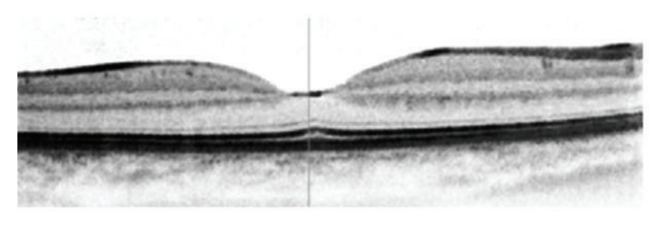

(c)

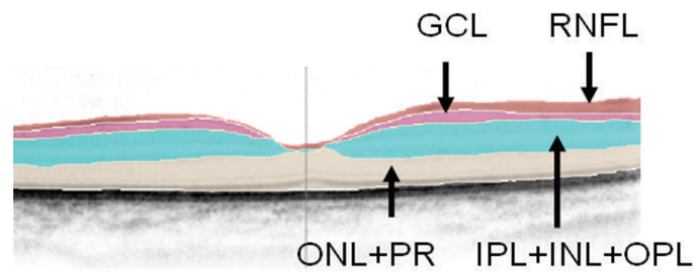

(d)

FIGURE 1: SDOCT scans with and without delineation of retinal boundaries for neurosensory retina, retinal nerve fiber layer (RNFL), ganglion cell layer (GCL), a combined space of inner and outer plexiform layers and inner nuclear layer (IPL+INL+OPL), and a combined space of the outer nuclear layer and photoreceptor layer $(\mathrm{ONL}+\mathrm{PR})$ : A+B: ADOA patient, $\mathrm{C}+\mathrm{D}$ : healthy control.

TABLE 1: Clinical and genetic details of ADOA patients.

\begin{tabular}{|c|c|c|c|c|c|c|c|}
\hline Case & Gender & OPA1 mutation & $\begin{array}{c}\text { Age at } \\
\text { examination } \\
\text { (years) }\end{array}$ & $\begin{array}{c}\text { Visual acuity } \\
\text { (Snellen) } \\
\text { OD/OS } \\
\end{array}$ & $\begin{array}{c}\text { Refraction (spherical } \\
\text { equivalent) } \\
\text { OD/OS } \\
\end{array}$ & Visual fields & Disc appearance \\
\hline 1 & $\mathrm{~m}$ & c1299_1305del12bp & 4 & $20 / 200 / 20 / 100$ & $+2.4 /+2.4$ & - & Temporal pallor \\
\hline 2 & $\mathrm{f}$ & $\begin{array}{l}\text { Duplication Exon } 1 \text { to } \\
4 \mathrm{~b} \text { of } O P A 1 \text { gene }\end{array}$ & 11 & $20 / 40 / 20 / 125$ & $-0.6 /-0.5$ & $\begin{array}{l}\text { Scotomas in the } \\
\text { upper temporal } \\
\text { fields }\end{array}$ & Temporal pallor \\
\hline 3 & $\mathrm{~m}$ & c.2614-9A>G & 18 & $20 / 60 / 20 / 60$ & $-2.0 /-1.5$ & $\begin{array}{l}\text { Scotomas in the } \\
\text { upper fields }\end{array}$ & Temporal pallor \\
\hline 4 & $\mathrm{f}$ & c.904A>C, p.Thr302Pro & 26 & $20 / 100 / 20 / 125$ & $-5.0 /-5.5$ & $\begin{array}{l}\text { Slight, unspecific } \\
\text { defects }\end{array}$ & Temporal pallor \\
\hline 5 & $\mathrm{~m}$ & $\begin{array}{c}\text { c.1979G > Ap.Trp660Stop } \\
\text { heterozygous }\end{array}$ & 28 & $20 / 60 / 20 / 60$ & $+0.5 /+1.0$ & $\begin{array}{l}\text { Scotomas OS }>\text { OD } \\
\text { especially in the } \\
\text { upper temporal } \\
\text { fields }\end{array}$ & Temporal pallor \\
\hline 6 & $\mathrm{~m}$ & $\begin{array}{c}\text { c.2708_2711 del TTAG } \\
\text { p.Val903GlyfsX3 } \\
\text { heterozygous }\end{array}$ & 43 & $20 / 125 / 20 / 100$ & $-2.0 /-2.9$ & $\begin{array}{l}\text { Scotomas in the } \\
\text { upper fields }\end{array}$ & $\begin{array}{l}\text { Pale disc, especially } \\
\text { temporally }\end{array}$ \\
\hline 7 & $\mathrm{f}$ & c. $2983+4 \quad A>G$ & 47 & $20 / 800 / 20 / 800$ & $-10.5 /-8.3$ & - & $\begin{array}{l}\text { Pale disc, especially } \\
\text { temporally }\end{array}$ \\
\hline
\end{tabular}

OD: right eye; OS: left eye.

reduction in peripapillary RNFL thickness with age in 10 ADOA patients with OPA1 mutations as well as in 30 healthy subjects using Stratus OCT. In ADOA patients, VA decreased significantly with age. Further, VA was correlated with peripapillary RNFL thickness in the inferior and superior peripapillary quadrants and with total macular thickness at eccentricities of 500-3000 $\mu \mathrm{m}$. In contrast, we did not neither find a significant correlations between RNFLs at the posterior pole and age nor between RNFLs at the posterior pole and
VA in our group. Differences in the individual course of the disease as well as our relatively small study group might be the reason for the lack in significant correlations with VA and age in our study.

Yu-Wai-Man et al. [9] recently also demonstrated a generalized decrease in peripapillary RNFL thickness on Stratus OCT in 40 patients with OPA1 mutations, with a statistically significant inverse correlation between RNFL thickness and $\log$ MAR VA. Additionally, they reported that the temporal 
TABLE 2: Median thickness values of retinal spaces in ADOA patients and healthy controls.

\begin{tabular}{lcccccccccc}
\hline \multirow{2}{*}{ Retinal space } & \multicolumn{2}{c}{$\begin{array}{c}\text { Subfield 5 } \\
(\mu \mathrm{m})\end{array}$} & \multicolumn{2}{c}{$\begin{array}{c}\text { Subfield 6 } \\
(\mu \mathrm{m})\end{array}$} & \multicolumn{2}{c}{$\begin{array}{c}\text { Subfield 7 } \\
(\mu \mathrm{m})\end{array}$} & \multicolumn{2}{c}{$\begin{array}{c}\text { Subfield 8 } \\
(\mu \mathrm{m})\end{array}$} & \multicolumn{2}{c}{$\begin{array}{c}\text { Inner circle } \\
(\mu \mathrm{m})\end{array}$} \\
& Patients & Controls & Patients & Controls & Patients & Controls & Patients & Controls & Patients & Controls \\
\hline Neurosensory retina & 259.7 & 311.2 & 259.7 & 314.0 & 256.5 & 312.5 & 258.4 & 299.8 & 259.9 & 310.1 \\
RNFL & 14.2 & 35.6 & 13.1 & 30.6 & 11.5 & 35.6 & 9.1 & 25.6 & 10.8 & 31.3 \\
GCL & 0.5 & 44.9 & 0.2 & 48.2 & 0.2 & 44.3 & 0.7 & 44.8 & 0.7 & 45.1 \\
IPL+INL+OPL & 124.7 & 126.7 & 124.4 & 124.0 & 128.1 & 122.9 & 123.0 & 115.0 & 126.1 & 123.6 \\
ONL+PL & 113.9 & 111.0 & 123.5 & 113.6 & 116.0 & 110.3 & 122.9 & 117.1 & 117.7 & 112.8 \\
\hline
\end{tabular}

RNFL: retinal nerve fiber layer; GCL: ganglion cell layer; INL: inner nuclear layer; IPL: inner plexiform layer; OPL: outer plexiform layer; ONL: outer nuclear layer; PL: photoreceptor layer.

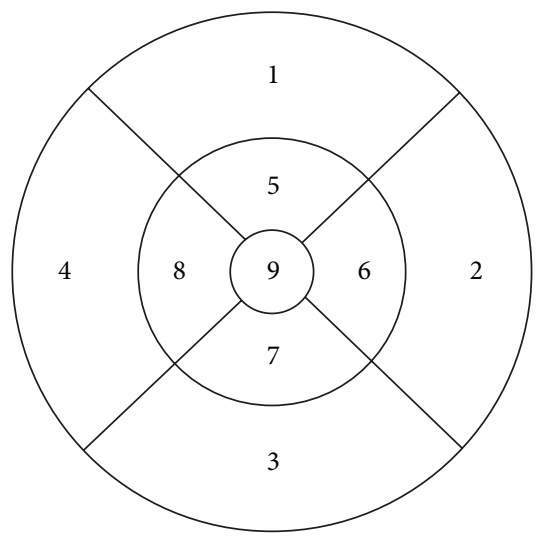

FIGURE 2: SDOCT thickness map with EDTRS inner circle (5-8) for OD: subfield 8: temporal, subfield 6: nasal.

quadrant was more severely affected and the nasal quadrant relatively spared, documenting the preferential involvement of the papillomacular bundle in ADOA patients.

These findings were confirmed by Barboni et al. [17], who compared peripapillary RNFL thickness of 33 patients to healthy controls using Stratus OCT.

Ito et al. [18] analyzed Stratus OCT images from the peripapillary region in 8 patients with OPA1 mutations. Additionally, the neurosensory retina was measured at the foveal center point and at two locations 1 and $2 \mathrm{~mm}$ temporal, nasal, superior, and inferior to the fovea for the following layers: RNFL, a "middle layer" including the GCL, IPL, INL, and OPL, a layer including the ONL and the photoreceptor inner segments, and the photoreceptor outer segment layer. The neurosensory retina in the macular area was found to be significantly thinner in patients with ADOA compared to healthy subjects at all points measured except for the fovea, and this was explained by a decrease in thickness of the combined GCL, IPL, INL, and OPL in the macular area.

Russo et al. [19] first used SDOCT images for analysis. Similar to Ito et al., they found a diffuse thinning of the combined space of RNFL, GCL, and IPL in sectional macular SDOCT scans of four affected family members with ADOA. Further, they confirmed the peripapillary RNFL thickness loss and found a correlation between average circumpapillary RNFL and VA.
As the papillomacular bundle was shown to be preferentially involved before $[8,9,17]$, we focused in our study on macular SDOCT volume scans. To allow for a more detailed subanalysis of various retinal layers, we used highresolution SDOCT images and manual grading software to calculate mean thickness values for various retinal spaces for the ETDRS subfields 5-8. We were able to measure the GCL separately and show that not only the RNFL but also the GCL is significantly reduced in ADOA patients compared to healthy controls, but IPL+INL+OPL thickness is not different to healthy subjects. This difference in RNFL and GCL was evident for the superior, inferior, nasal, and temporal subfield of the ETDRS grid. In healthy controls subfield 8 (temporal) showed the thinnest retinal thickness, INL+IPL+OPL and RNFL values compared to the other subfields. For RNFL, this difference was even evident in ADOA patients in our group.

Beside several strengths, namely, the use of SDOCT and analysis of different retinal layers manually delineated on each $B$-scan, our study also has limitations such as the small study group and the inclusion of children despite the lack of a normative database in the young.

\section{Conclusions}

In conclusion, manual subanalysis of high-resolution macular SDOCT volume scans revealed that not only RNFL thickness but also GCL thickness is reduced in ADOA patients in subfields $5-8$, whereas there is no significant difference in IPL+INL+OPL thickness. For patients with loss of vision of unclear origin, SDOCT helps to distinguish between disorders of the outer retinal layers and disorders of the inner retinal layers, for example, optic neuropathies. Together with clinical history and examination, SDOCT may help to identify patients with suspicion for hereditary optic neuropathies before genetic analysis confirms the diagnosis. This technique may further be important for the documentation of possible effects of future therapies like gene therapy, in addition to clinical parameters like measurement of VA and visual fields.

\section{Authors' Contribution}

Andrea M. Schild and Tina Ristau equally contributed as first authors. 


\section{References}

[1] M. Ferré, D. Bonneau, D. Milea et al., "Molecular screening of 980 cases of suspected hereditary optic neuropathy with a report on 77 novel OPA1 mutations," Human Mutation, vol. 30, no. 7, pp. E692-E705, 2009.

[2] N. Fuhrmann, M. V. Alavi, P. Bitoun et al., "Genomic rearrangements in OPAl are frequent in patients with autosomal dominant optic atrophy," Journal of Medical Genetics, vol. 46, no. 2, pp. 136-144, 2009.

[3] C. Frezza, S. Cipolat, O. Martins de Brito et al., "OPA1 controls apoptotic cristae remodeling independently from mitochondrial fusion," Cell, vol. 126, no. 1, pp. 177-189, 2006.

[4] A. Olichon, E. Guillou, C. Delettre et al., "Mitochondrial dynamics and disease, OPA1," Biochimica et Biophysica Acta, vol. 1763, no. 5-6, pp. 500-509, 2006.

[5] D. Eliott, E. I. Traboulsi, and I. H. Maumenee, "Visual prognosis in autosomal dominant optic atrophy (Kjer type)," American Journal of Ophthalmology, vol. 115, no. 3, pp. 360-367, 1993.

[6] P. Kjer, "Infantile optic atrophy with dominant mode of inheritance: a clinical and genetic study of 19 Danish families," Acta Ophthalmologica, vol. 164, supplement 54, pp. 1-147, 1959.

[7] M. Votruba, D. Thiselton, and S. S. Bhattacharya, "Optic disc morphology of patients with OPAl autosomal dominant optic atrophy," British Journal of Ophthalmology, vol. 87, no. 1, pp. 4853, 2003.

[8] P. Kjer, O. A. Jensen, and L. Klinken, "Histopathology of eye, optic nerve and brain in a case of dominant optic atrophy," Acta Ophthalmologica, vol. 61, no. 2, pp. 300-312, 1983.

[9] P. Yu-Wai-Man, M. Bailie, A. Atawan, P. F. Chinnery, and P. G. Griffiths, "Pattern of retinal ganglion cell loss in dominant optic atrophy due to OPA1 mutations," Eye, vol. 25, no. 5, pp. 596-602, 2011.

[10] S. S. Choi, R. J. Zawadzki, J. L. Keltner, and J. S. Werner, "Changes in cellular structures revealed by ultra-high resolution retinal imaging in optic neuropathies," Investigative Ophthalmology and Visual Science, vol. 49, no. 5, pp. 2103-2119, 2008.

[11] J. Pasol, "Neuro-ophthalmic disease and optical coherence tomography: glaucoma look-alikes," Current Opinion in Ophthalmology, vol. 22, no. 2, pp. 124-132, 2011.

[12] S. Srinivasan, U. K. Addepalli, H. L. Rao, C. S. Garudadri, and A. K. Mandal, "Spectral domain optical coherence tomography in children operated for primary congenital glaucoma," British Journal of Ophthalmology, 2013.

[13] S. R. Sadda, S. Joeres, Z. Wu et al., "Error correction and quantitative subanalysis of optical coherence tomography data using computer-assisted grading," Investigative Ophthalmology and Visual Science, vol. 48, no. 2, pp. 839-848, 2007.

[14] S. Joeres, J. W. Tsong, P. G. Updike et al., "Reproducibility of quantitative optical coherence tomography subanalysis in neovascular age-related macular degeneration," Investigative Ophthalmology and Visual Science, vol. 48, no. 9, pp. 4300-4307, 2007.

[15] F. M. Heussen, Y. Ouyang, E. C. McDonnell et al., "Comparison of manually corrected retinal thickness measurements from multiple spectral-domain optical coherence tomography instruments," British Journal of Ophthalmology, vol. 96, no. 3, pp. 380-385, 2012.

[16] D. Milea, B. Sander, M. Wegener et al., "Axonal loss occurs early in dominant optic atrophy," Acta Ophthalmologica, vol. 88, no. 3, pp. 342-346, 2010.
[17] P. Barboni, G. Savini, V. Parisi et al., "Retinal nerve fiber layer thickness in dominant optic atrophy: measurements by optical coherence tomography and correlation with age," Ophthalmology, vol. 118, no. 10, pp. 2076-2080, 2011.

[18] Y. Ito, M. Nakamura, T. Yamakoshi, J. Lin, H. Yatsuya, and H. Terasaki, "Reduction of inner retinal thickness in patients with autosomal dominant optic atrophy associated with OPA1 mutations," Investigative Ophthalmology and Visual Science, vol. 48, no. 9, pp. 4079-4086, 2007.

[19] A. Russo, L. Delcassi, E. Marchina, and F. Semeraro, "Correlation between visual acuity and OCT-measured retinal nerve fiber layer thickness in a family with ADOA and an OPA1 mutation," Ophthalmic Genetics, vol. 34, no. 1-2, pp. 69-74, 2012. 


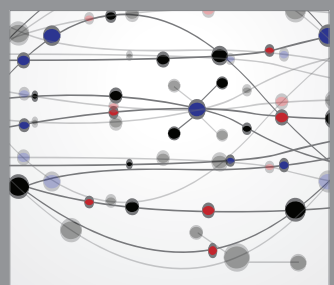

The Scientific World Journal
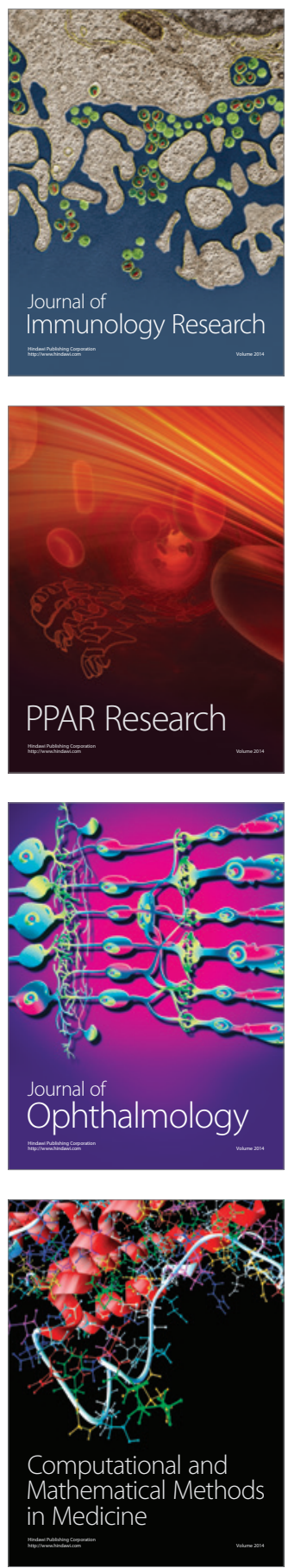

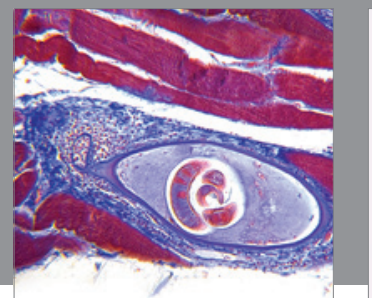

Gastroenterology

Research and Practice
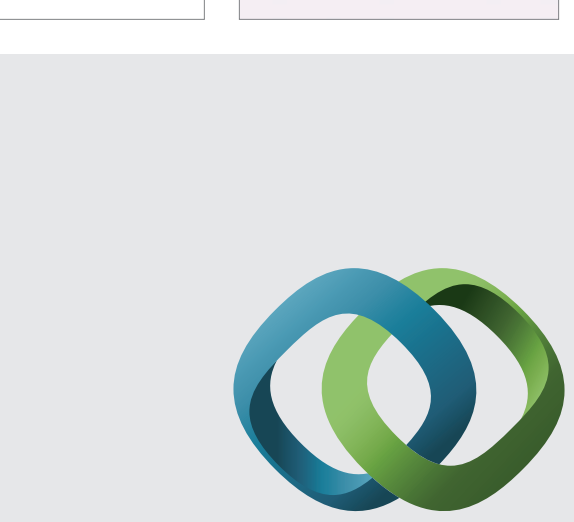

\section{Hindawi}

Submit your manuscripts at

http://www.hindawi.com
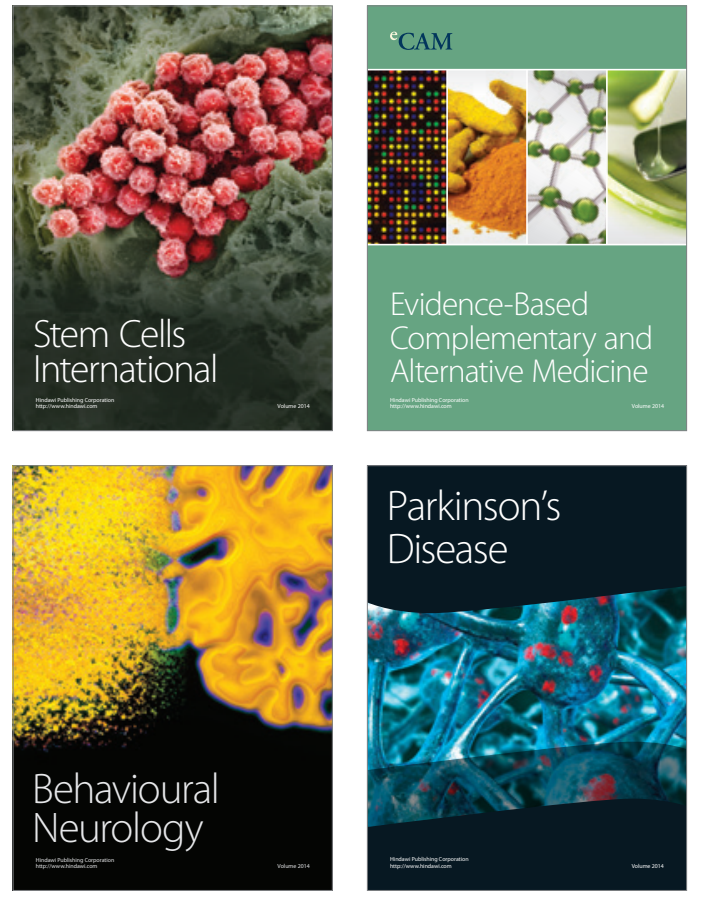
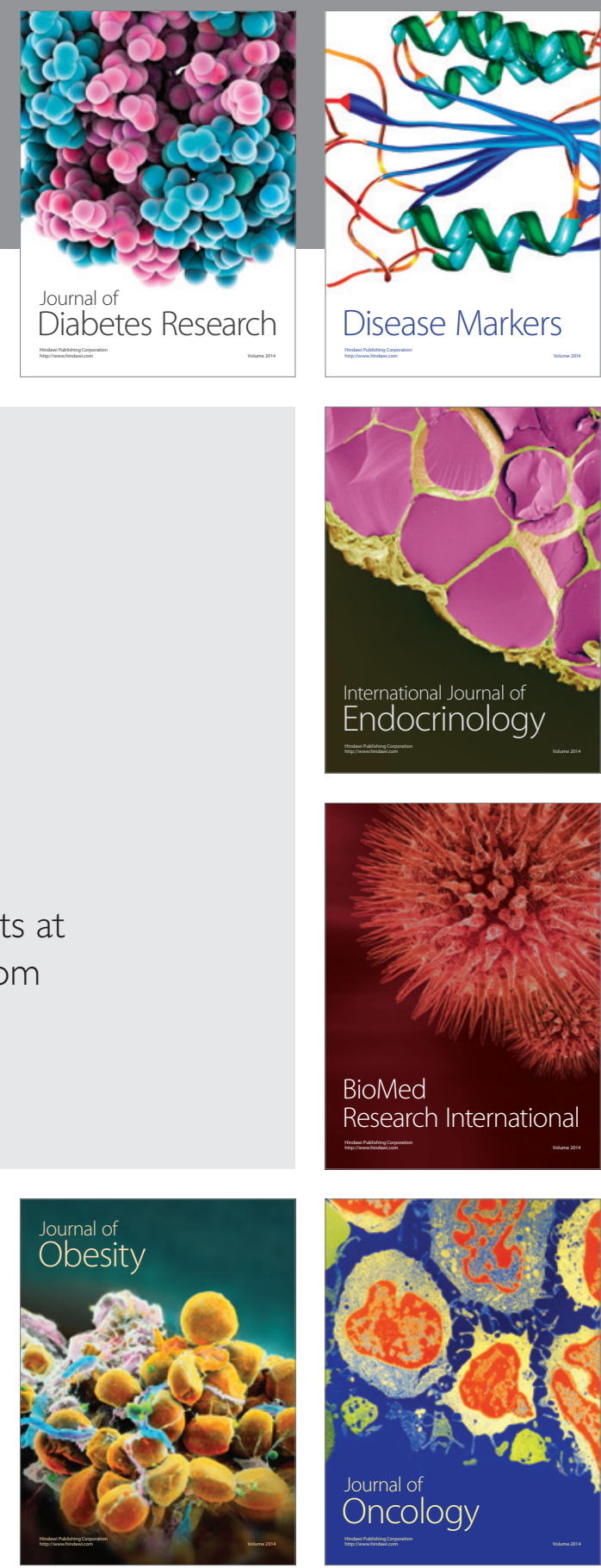

Disease Markers
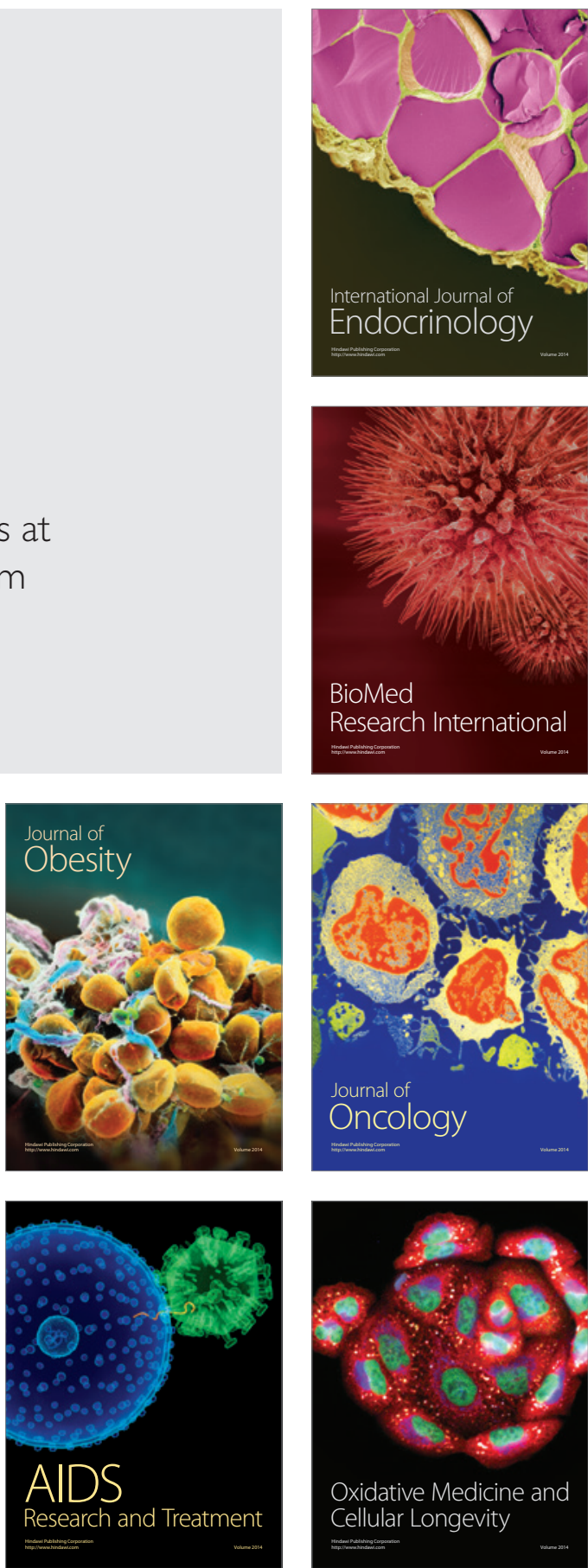